\title{
Neither Black nor White? An empirical test of the Latin Americanization thesis
}

\author{
Tyrone A. Forman ${ }^{\mathrm{a}, \mathrm{b}, *}$, Carla Goar ${ }^{\mathrm{c}}$, Amanda E. Lewis ${ }^{\mathrm{a}}$ \\ ${ }^{a}$ Departments of Sociology and African American Studies, Institute for Research on Race and Public Policy, \\ 1007 West Harrison Street, Chicago, IL 60607, USA \\ ${ }^{\mathrm{b}}$ Institute of Government and Public Affairs, University of Illinois at Chicago, \\ 815 West Van Buren Street, Chicago, IL 60607, USA \\ ${ }^{\mathrm{c}}$ Department of Sociology, Northern Illinois University, DeKalb, IL 60115, USA
}

\begin{abstract}
Over the past four decades, demographic trends have provoked divergent perspectives concerning the future of race and ethnic relations in the United States. Despite the important implications of these differing interpretations of the future, social scientists have given them little empirical scrutiny. In this study, we systematically investigate one of these perspectives-Bonilla-Silva et al. [Research in Political Sociology 23 (2003) 111] Latin Americanization thesis. The Latin Americanization thesis posits that the United States is moving from a two-tier, White/non-White, racial stratification system toward a three-tier one that consists of the following categories: (1) White, (2) honorary White, and (3) collective Black. In this article, we empirically test one aspect of this thesis - the idea that members of the "White," "honorary White" and "collective Black" categories will express social attitudes that reflect their status position in the newly emergent three-tier racial stratification system. We use a large nationally representative sample of non-Hispanic Whites, non-Hispanic Blacks, Mexicans, Puerto Ricans, and Cubans to assess this question. Our results lend qualified support to the Latin Americanization thesis. We conclude by considering the implications of these results for future research on contemporary racial dynamics.
\end{abstract}

(C) 2004 Elsevier Inc. All rights reserved.

Keywords: Latin Americanization thesis; Racial dynamics; Latinos; Racial attitudes

The population of people of color in the United States is rapidly expanding. Indeed, demographers predict that Americans of color will comprise a numerical majority no later than 2050 (Farley, 1996; McDaniel, 1995). Much of this expansion is the direct result of massive

${ }^{*}$ Corresponding author. Tel.: +1-312-996-2915.

E-mail addresses: goar@niu.edu, tyforman@uic.edu (T.A. Forman), aelewis@ uic.edu (A.E. Lewis). 
immigration from Latin America and Asia. Despite the substantial growth in the Latino and Asian-American populations, research on race relations in the United States continues to be dominated by a binary Black-White paradigm (Almaguer, 1994; Bonilla-Silva, Forman, Lewis, \& Embrick, 2003; McKee, 1993). Not only has this dualist perspective limited the empirical investigation of competing visions of the future of U.S. race relations, but it has also produced limited research on specific populations, including Latinos. ${ }^{1}$ This scholarly inattention is increasingly problematic because Latinos are not only the fastest growing segment of the U.S. population, increasing by 58\% between 1990 and 2000, but also the largest racial/ethnic minority group (U.S. Census Bureau, 2001).

Though not receiving sufficient attention, shifts in the population have not gone entirely unnoticed by analysts of race and ethnic relations. These demographic patterns have provoked some new theorizing including several interesting and divergent predictions concerning the future of race and ethnic relations in the United States. One perspective claims that the future of U.S. race relations will remain a binary with shifts only in the parameters of the two dominant categories or in who belongs in which category (Fields, 2001; Gans, 1999; Lind, 1998; Warren \& Twine, 1997; Yancey, 2003). For example, Yancey (2003) argues that the primary divide will go from one that historically has been between Whites and non-Whites to one between Blacks and non-Blacks. He notes, "the White/non-White dichotomy currently used to understand race relations will eventually need to be replaced with a Black/non-Black dichotomy. Instead of evaluating the social acceptance of a group by how 'White' they are, it will be more important to assess the social rejection of a group by how 'Black' they are" (Yancey, 2003, p. 15). In this case Blacks would remain at the bottom of the racial/ethnic hierarchy with the primary division being between Blacks (as the primary have-nots) and all others rather than between Whites (as the primary haves) and all others (for a similar perspective see Gans, 1999). In a related but slightly different vein, Warren and Twine (1997) have suggested that the primary transformation that will take place is the expansion of the social category of "White." They argue that rather than diminishing in relative size as a result of increasing numbers of Asians and Latinos, the category "White" may well expand to incorporate new groups in the same way it did at the beginning of the 20th century (e.g., Italians, Irish, Polish). ${ }^{2}$ As Yancey (2003, p. 3) put it, "the current predictions about Whites becoming a numerical minority are wrong not because of incorrect assessments of the growth of racial minorities, but because the definition of who is White is not static." According to Warren and Twine (1997) then, our notions of who is "White" will shift, incorporating many Latinos and Asians into the "White" category with "Whites" thus remaining a numerical majority.

A second perspective on the future of race and ethnic relations in the United States suggests that the traditional binary will no longer exist. Bonilla-Silva et al. (2003) argue that race relations in the United States will follow a similar pattern observed in several Latin American and Caribbean nations (thus, the name, Latin Americanization). That is, increasingly both the racial discourse and the racial and ethnic stratification system of the United States will resemble the much more complex patterns observed in Latin American countries such as Brazil, Belize, Columbia, Cuba, and Mexico (see Degler, 1971; Rodriguez, 2000). As they outline it, at least a three-tier racial divide will best characterize the future of U.S. race relations. In this racial hierarchy, while a traditional "White"3 category remains at the top of racial/ethnic 
hierarchy, some Asians and some Latinos are likely to be placed into a newly emerging middle category of "honorary White." This middle category will serve an important buffering function between "White" and what Bonilla-Silva et al. (2003) call "the collective Black." This third category includes not only African-Americans, but some groups of Latinos and Asians (e.g., dark-skinned Puerto Ricans and Dominicans, Cambodians and Laotians). This perspective builds off and attempts to account for social indicators such as current patterns of residential segregation (e.g., in which, for instance, non-Hispanic Whites are much more residentially integrated with some groups of Asians and Latinos than they are with Blacks and in which dark-skinned Latinos are much more integrated with Blacks than with Whites; see Freeman, 1999; Massey \& Fischer, 1999) ${ }^{4}$ as well as racial attitudes in which, for instance, Whites express preference for some racial minorities over others (e.g., in a recent study a White mother responded to the question, "Do you think it would be a problem for you or your husband if your daughter or your son married someone from a different race?" by stating that it would be okay with her if they married an Asian person but not a Black person; see Lewis, 2003, pp. 23-24).

This perspective is consistent with recent theorizing on contemporary immigrant assimilation. Though addressing patterns of immigration rather than race relations overall, recent writing about "segmented assimilation" (Portes \& Zhou, 1993; Zhou, 1997) similarly posits three possible outcomes for new immigrants to the U.S.—-roughly representing those who assimilate completely and seamlessly into the dominant culture, those who represent a middle category, and those who become "assimilated" into the lowest rungs of American society. Challenging the notion of a monolithic immigrant experience which assumes a linear progression of adaptation to the mainstream of the host society (see Alba \& Nee, 1997; Gordon, 1964; Suarez-Orozco, 2000), segmented assimilation theorists suggest that there are multiple outcomes for post-1965 immigrants: (1) acculturation and integration into White society, (2) preservation of cultural traditions and social networks from country of origin, and (3) acculturation and integration into the impoverished lower stratum of U.S. society (Zhou, 1997). ${ }^{5}$

Despite the important implications that these differing interpretations have for understanding contemporary racial dynamics, in general, social scientists have given them little empirical scrutiny. Therefore, in this article we systematically investigate one of these perspectivesBonilla-Silva et al. (2003) Latin Americanization thesis. Specifically we focus on one aspect of their thesis, the notion that members of "White," "honorary White" and "collective Black" categories will express social attitudes that reflect their status position in the newly emergent racial stratification system. We argue that our focus on attitudes in this study is important because race relations are shaped not only by the differing structural location of racial and ethnic groups, but also by attitudes and beliefs. Individual's racial attitudes are not independent of the societal context in which they are embedded. Stable social structures of racial and ethnic inequality condition and nurture the expression of racial attitudes in multiple mesoand micro-level contexts (see Forman, 2001). Thus, racial attitudes are not merely negative attitudes held by one individual against another but also reflect the social structural relations between racial groups. As such, an analysis of possible cleavages in racial attitudes is central to understanding how race relations are currently structured and will likely evolve in the future. 


\section{The Latin Americanization thesis}

Bonilla-Silva et al. (2003) develop the "Latin Americanization thesis" to describe the future of race relations in the United States. They posit that the demographic trends that are altering the U.S. racial landscape are not unique, but reflect a pattern of change that occurred earlier (during the 16th, 17th, 18th and 19th centuries) in several Latin American and Caribbean countries. As it pertains to the racial and ethnic stratification system they suggest that the two-tier system of "White/non-White" will evolve into a new three-tier system. This tri-racial stratification system has three core groups: “ 'Whites' at the top, an intermediary group of 'honorary Whites' (similar to the coloreds in South Africa during formal apartheid) and a non-White group or the "collective Black' at the bottom" (Bonilla-Silva et al., 2003, p. 121). According to their thesis the "White" category will be composed of "traditional" Whites such as the English, Germans, Italians, Irish, Polish, new White immigrants such as Russians, Albanians, Serbians, and Croatians, as well as assimilated Latinos such as Argentines and Cubans who arrived prior to 1980 (see Fig. 1). ${ }^{6}$ The "honorary White" category consists of light-skinned Latinos, Japanese, Chinese, Koreans, and some multi-racial individuals. Finally, the "collective Black" category will include U.S. born Blacks, many immigrants and second-generation individuals from the Caribbean (e.g., Haiti, Jamaica), African immigrants, dark-skinned Latinos (e.g., many Dominicans and Puerto Ricans as well as Cubans who arrived in 1980 and $\operatorname{after}^{7}$ ), Cambodians, Laotians, Hmong, Filipinos, and reservation based Native Americans.

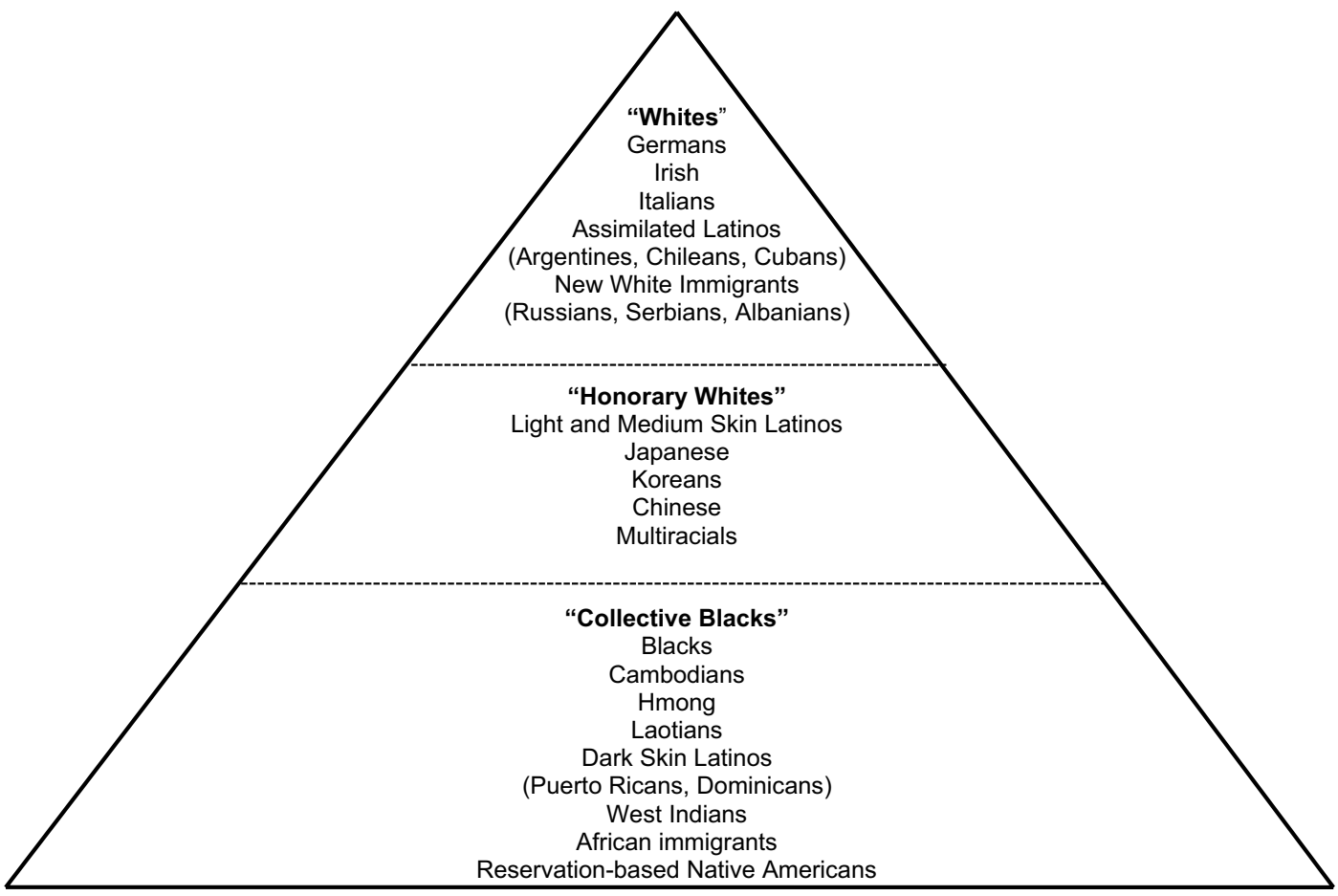

Fig. 1. Three-tier racial hierarchy in the United States. 
There are several features to this three-tier Latin-American-like racial stratification system. First, explicit Black-White conflict will be reduced through a process of diffusion as the presence of intermediate groups complicates traditional racial hierarchies and tensions. Second, new forms of "shade discrimination" will dominate the three-tier stratification system. ${ }^{8}$ Third, this more nuanced and complex system will be accompanied by a new racial discourse. For example, regarding the changing racial discourse Bonilla-Silva et al. (2003, p. 121) note, "the United States will begin to exhibit the ostrich approach to racial matters of countries [in Latin America] that stick their head deep into the social ground and say, 'We don't have races here. We don't have racism here. We are all Mexicans, Cubans, Brazilians, or Puerto Rican!' ' Thus, though racial and ethnic inequality will persist, large numbers of U.S. residents will increasingly espouse nationalist appeals such as "we are all American" and simultaneously claim to live in a post-race, color-blind world where racial and ethnic disparities are no longer the result of discrimination (Bonilla-Silva et al., 2003; Forman, in press). This new racial discourse along with reduced Black-White conflict will reinforce Blacks social location at the bottom of the U.S. racial hierarchy. In short, despite expansion to a multi-polar racial hierarchy in the U.S. as a result of increasing numbers of Asians and Latinos, anti-blackness will remain the lynchpin of White supremacy. ${ }^{9}$ As philosopher Lewis Gordon (1997, p. 57) highlights anti-blackness "persistence suggests that the fluidity of racial identities points upward in continuing spirals of potential whiteness."

\section{The present study}

According to Bonilla-Silva et al. (2003), if a three-tier racial stratification system is emerging in the United States, one would expect individuals to express racial attitudes that reflect their location in the racial order. For instance, among Whites we might expect an emphasis on retaining overall privilege while simultaneously maintaining a pragmatic relationship with members of the honorary White group (based on mutual distaste of members of the collective Black). Honorary White group members may exaggerate their similarity with Whites while distancing themselves from the collective Black. Membership in this group is precarious as the threat of being demoted to collective Black status is ever present (e.g., Middle Easterners). Collective Black members may strive to align themselves with sympathetic honoraries or create intragroup hierarchies based on other characteristics (i.e., skin tone). Here we are interested in two aspects of the thesis. First, whether there are in fact intragroup differences in attitudes toward social issues associated with skin color. Second, whether and how different Latino ethnic groups seem to fit into a three-tier racial hierarchy. Bonilla-Silva et al. (2003) initially place Latinos into three different categories within their model-largely based on skin color. We examine whether there is in fact a pattern of skin color differentiation in which some Latinos seem to fit into an emergent middle category of "honorary White" while others' social attitudes seem to align better with Whites or with Black. Or is the pattern one in which ethnicity seems to play more of a role in shaping attitudes? To explore these aspects of the Latin Americanization thesis we use a large nationally representative sample of non-Hispanic Whites, non-Hispanic Blacks, Mexicans, Puerto Ricans, and Cubans. ${ }^{10}$ 


\section{Data and measures}

\subsection{Sample}

We use data from the 1990 Latino National Political Survey (LNPS), a national sample of 3,415 Latino, non-Latino White, and non-Latino Black respondents. The design and methods of the study are summarized briefly here (a more detailed description is available elsewhere: see de la Garza, DeSipio, Garcia, Garcia, \& Falcon, 1992; de la Garza, Falcon, \& Garcia, 1996). The LNPS is a multistage area probability sample representative of the Latino population 18 years of age or older, residing in the 48 conterminous states and the District of Columbia. The LNPS included non-Hispanic Whites $(n=468)$ and Blacks $(n=80)$ as well as the three largest Latino subgroups in the United States: Mexicans $(n=1,546)$, Puerto Ricans $(n=589)$, and Cubans $(n=682)$. These three groups accounted for approximately three quarters $(77 \%)$ of the Latino population in 1990. Face-to-face interviews were completed between July 1989 and March 1990 by professional interviewers from the Institute for Survey Research at Temple University. Interviews with Latino respondents were conducted in either English or Spanish based upon their preference (60\% of the interviews were conducted in Spanish). Interviews conducted in English averaged 83 minutes, while those in Spanish averaged 91 minutes. The Latino response rate was $82 \%$ while the non-Latino response rate was $62 \%$. All analyses use sample weights to take into account differential probabilities of selection, nonresponse bias, and poststratification adjustments to the sample.

\subsection{Dependent variables}

We use three indicators of social attitudes in this study: attitudes toward increased government assistance to help specific groups, attitudes toward perceived group discrimination, and a feeling thermometer measuring attitudes toward specific groups. All ordinal and interval scaled measures were keyed so that a high score reflects a high value of the construct.

\subsubsection{Attitudes toward increased government assistance}

Each respondent was read the following statement: Now we would like to ask you about your views on various types of government programs. As I read each program, tell me if you would like to see it increased even if it meant paying more taxes, if you would like to see it decreased or if you would leave it the same. How about programs to help legal immigrants and refugees? How about programs to help Blacks? Possible responses ranged from "increase" (3), "left the same" (2), or "decrease" (1). ${ }^{11}$

\subsubsection{Extent of group discrimination}

Each respondent was asked the following question concerning the extent of discrimination: Do you think Blacks face a lot of discrimination, some, a little, or no discrimination at all? Possible responses ranged from "a lot" (4) to "none" (1).

\subsubsection{Feeling thermometer}

Each respondent was asked to indicate on a feeling thermometer scale ranging from 0 to 100 how "warm" or "cold" they felt toward Whites and Blacks. A score of 0 reflects 
a very cold feeling, a score of 50 reflects a neutral feeling, and a score of 100 reflects a very warm feeling. At first glance, these measures may appear to be susceptible to severe social desirability effects; however, these measures were self-administered and previous research indicates that self-administered racial attitude questions typically reduce social desirability pressures (see Jackman \& Crane, 1986; Krysan, 1998). Further, the feeling thermometer scale has been used extensively in previous research (see Bobo \& Hutchings, 1996; Sears, Van Laar, Carrillo, \& Kosterman, 1997; Welch \& Sigelman, 2000). Responses were keyed so that a high score indicates positive affect toward the target group (i.e., Whites and Blacks).

\subsection{Independent variables}

Our key independent variables are race/ethnicity (Mexican, Puerto Rican, Cuban, White, and Black) and skin color (determined by interviewer and coded as "1" dark, "2" medium, "3" light). The control variables used in this article were selected based upon previous research. We include a number of social background characteristics including: gender $(1=$ male $)$, age, education (years of formal education), income (ranging from " 1 ” (0 to $\$ 4,999)$ to " 15 " (\$75,000 or more)), nativity (born in the United States, $1=$ yes), and acculturation (percent of life lived in the United States). ${ }^{12}$ Three variables measure political orientation: party identification, political ideology, and love for the United States. Political ideology was measured on a 7-point scale ranging from " 1 " (extremely liberal) to " 7 " (extremely conservative). Party identification was coded: Republican, Democrat, Independent, No preference, and Don't know. Love for the United States was measured from responses to the following question: "How strong is your love for the United States?" Possible responses ranged from "extremely strong" (4) to "not very strong" (1).

\subsection{Analysis strategy}

Our major goal in this study is to answer the following question: Do Mexicans, Puerto Ricans, and Cubans of various skin colors express attitudes toward race-related issues that are similar to Whites or Blacks, or do they seem to occupy a distinct middle category as honorary Whites? To address this question, our analyses proceed in three stages. First, we present descriptive statistics exploring the bivariate association between Latino ethnicity and racial self-classification as well as interviewer assessment of skin tone (Figs. 2 and 3). Second, we explore the bivariate relationship between race-related attitudes and race/ethnicity and skin tone (Table 1). Finally, our multivariate analyses consist of a three-step ordinary least squares (OLS) regression analysis (Tables 2 and 3). ${ }^{13}$ For each dependent variable, we first estimate a baseline model of the gross (zero-order) effect of race/ethnicity and skin tone on our dependent variables (Model 1). Next, Model 2 reports these coefficients net of controls for social background factors such as age, gender, income, education, nativity and acculturation. Finally, Model 3 shows these coefficients after controlling for several measures of political orientation. We also examined each regression model for evidence of multicollinearity and influential observations. Our robustness tests indicate little evidence of a problematic model specification. 


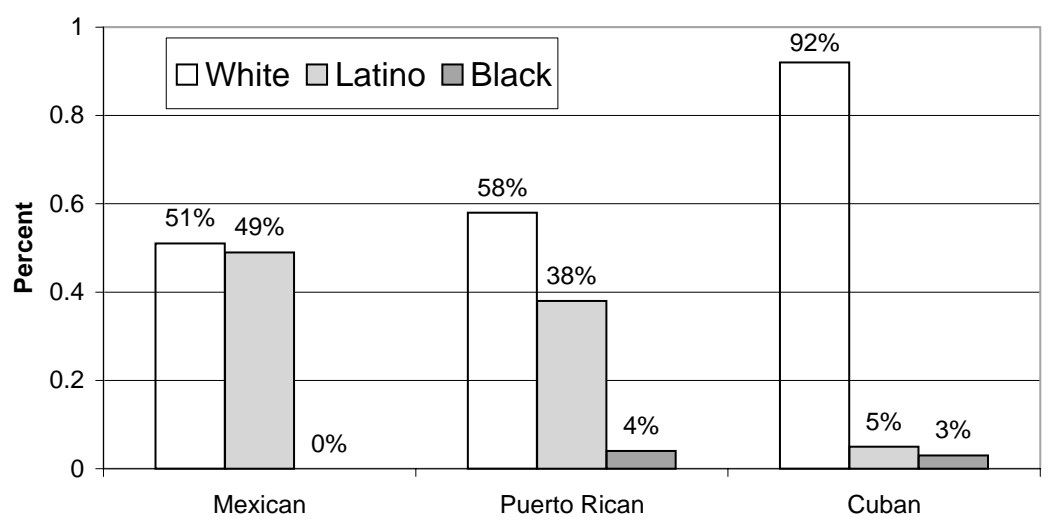

Fig. 2. Racial self-classification by Latino ethnicity.

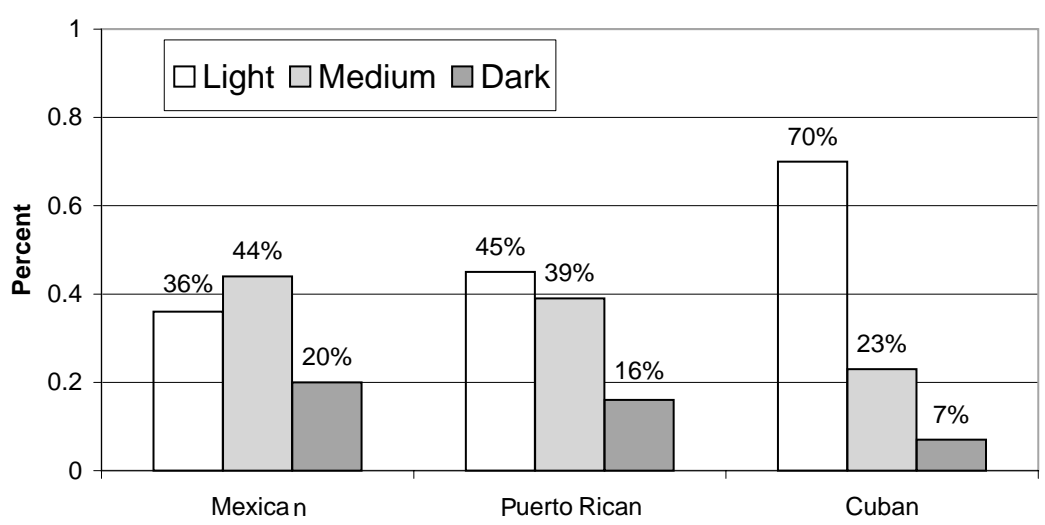

Fig. 3. Skin color classification by Latino ethnicity.

\section{Findings}

Does racial self-classification vary by Latino ethnicity? Fig. 2 indicates that although a majority of Mexicans, Puerto Ricans, and Cubans self-identify as "White" there are important ethnic differences. For instance, 9 in 10 Cubans, compared with 6 in 10 Puerto Ricans and 5 in 10 Mexicans chose the "White" category. A substantial minority of Mexican and Puerto Ricans (49 and 38\%, respectively) rejected the Census Bureau's racial classification of "Black" and "White" and chose instead the "Latino" category (for similar results see Rodriguez, 1992, 2000; Rodriguez \& Cordero-Guzman, 1992). It is interesting to note that very few Latinosonly 4\% of Puerto Ricans and 3\% of Cubans-self-identify as "Black." Race and skin color are obviously quite complex and do not map onto each other neatly. While many Latinos identity as "White" and few as "Black," interviewer assessments of skin color were somewhat different.

As shown in Fig. 3, based on the interviewers' assessment of the respondents' skin color, $20 \%$ of Mexicans, $16 \%$ of Puerto Ricans, and $7 \%$ of Cubans were labeled as having a dark 
Table 1

Race-related attitudes by race/ethnicity and skin tone: 1990 National Latino Political Survey

\begin{tabular}{|c|c|c|c|c|c|c|c|c|c|c|c|c|}
\hline & \multirow[b]{2}{*}{ Blacks } & \multicolumn{3}{|c|}{ Dark complexion } & \multicolumn{3}{|c|}{ Medium complexion } & \multicolumn{3}{|c|}{ Light complexion } & \multirow[b]{2}{*}{ Whites } & \multirow[b]{2}{*}{$X^{2} / F$ ratio } \\
\hline & & Mexcians & $\begin{array}{l}\text { Puerto } \\
\text { Ricans }\end{array}$ & Cubans & Mexicans & $\begin{array}{l}\text { Puerto } \\
\text { Ricans }\end{array}$ & Cubans & Mexicans & $\begin{array}{l}\text { Puerto } \\
\text { Ricans }\end{array}$ & Cubans & & \\
\hline \multicolumn{13}{|c|}{ Increase government programs to help (\%) } \\
\hline Blacks & 54.3 & 61.1 & 64.6 & 69.6 & 58.2 & 72.9 & 48.4 & 58.5 & 63.6 & 41.1 & 24.1 & $309.87^{* *}$ \\
\hline Legal immigrants and refugees & 29.1 & 63.5 & 59.6 & 82.6 & 57.2 & 60.9 & 63.6 & 56.3 & 50.6 & 55.4 & 19.1 & $426.38^{* *}$ \\
\hline \multicolumn{13}{|c|}{ A lot of discrimination against (\%) } \\
\hline Blacks & 62.5 & 41.9 & 59.4 & 32.6 & 37.9 & 59.6 & 20.8 & 38.3 & 54.4 & 26.1 & 32.3 & $432.11^{* *}$ \\
\hline \multicolumn{13}{|c|}{ Degrees on feeling thermometer $\left(0-100^{\circ}\right)$} \\
\hline Whites & 64.94 & 69.83 & 66.44 & 72.89 & 70.07 & 65.48 & 71.26 & 73.96 & 70.57 & 76.50 & 78.62 & $9.91^{* *}$ \\
\hline Blacks & 84.04 & 61.47 & 57.68 & 55.63 & 58.28 & 61.99 & 57.57 & 61.64 & 62.89 & 56.65 & 62.84 & $10.74^{* *}$ \\
\hline
\end{tabular}

${ }^{* *} p \leq .01$ (two-tailed tests). 
Table 2

Gross and net effect of race/ethnicity and skin color on government help for Blacks and legal immigrants (standard errors in parentheses)

\begin{tabular}{|c|c|c|c|c|c|c|}
\hline & \multicolumn{3}{|c|}{ Government help Blacks } & \multicolumn{3}{|c|}{ Government help legal immigrants } \\
\hline & Model 1 & Model 2 & Model 3 & Model 1 & Model 2 & Model 3 \\
\hline \multicolumn{7}{|l|}{ Race/ethnicity } \\
\hline Blacks & $\begin{array}{c}0.638^{* *} \\
(0.118)\end{array}$ & $\begin{array}{l}0.617^{\text {** }} \\
(0.117)\end{array}$ & $\begin{array}{l}0.576^{\text {** }} \\
(0.118)\end{array}$ & $\begin{array}{r}0.174 \\
(0.123)\end{array}$ & $\begin{array}{r}0.147 \\
(0.123)\end{array}$ & $\begin{array}{r}0.158 \\
(0.123)\end{array}$ \\
\hline Dark Mexicans & $\begin{array}{l}0.442^{* *} \\
(0.055)\end{array}$ & $\begin{array}{l}0.374^{* *} \\
(0.057)\end{array}$ & $\begin{array}{l}0.352^{* *} \\
(0.057)\end{array}$ & $\begin{array}{l}0.654^{* *} \\
(0.057)\end{array}$ & $\begin{array}{l}0.538^{* *} \\
(0.060)\end{array}$ & $\begin{array}{l}0.520^{* *} \\
(0.060)\end{array}$ \\
\hline Dark Puerto Ricans & $\begin{array}{l}0.519^{* *} \\
(0.076)\end{array}$ & $\begin{array}{l}0.457^{* *} \\
(0.076)\end{array}$ & $\begin{array}{l}0.428^{* *} \\
(0.077)\end{array}$ & $\begin{array}{l}0.669^{* *} \\
(0.080)\end{array}$ & $\begin{array}{l}0.576^{* *} \\
(0.080)\end{array}$ & $\begin{array}{l}0.540^{* *} \\
(0.081)\end{array}$ \\
\hline Dark Cubans & $\begin{array}{l}0.578^{* *} \\
(0.103)\end{array}$ & $\begin{array}{l}0.513^{* *} \\
(0.103)\end{array}$ & $\begin{array}{l}0.507^{* *} \\
(0.103)\end{array}$ & $\begin{array}{l}0.898^{* *} \\
(0.108)\end{array}$ & $\begin{array}{l}0.804^{* *} \\
(0.108)\end{array}$ & $\begin{array}{l}0.804^{* *} \\
(0.108)\end{array}$ \\
\hline Medium Mexicans & $\begin{array}{l}0.407^{* *} \\
(0.048)\end{array}$ & $\begin{array}{l}0.354^{* *} \\
(0.050)\end{array}$ & $\begin{array}{l}0.339^{* *} \\
(0.050)\end{array}$ & $\begin{array}{l}0.534^{* *} \\
(0.050)\end{array}$ & $\begin{array}{l}0.430^{* *} \\
(0.052)\end{array}$ & $\begin{array}{l}0.415^{* *} \\
(0.052)\end{array}$ \\
\hline Medium Puerto Ricans & $\begin{array}{l}0.519^{* *} \\
(0.059)\end{array}$ & $\begin{array}{l}0.446^{* *} \\
(0.060)\end{array}$ & $\begin{array}{l}0.412^{* *} \\
(0.061)\end{array}$ & $\begin{array}{l}0.624^{* *} \\
(0.061)\end{array}$ & $\begin{array}{l}0.515^{* *} \\
(0.063)\end{array}$ & $\begin{array}{l}0.499^{* *} \\
(0.064)\end{array}$ \\
\hline Medium Cubans & $\begin{array}{l}0.305^{* *} \\
(0.066)\end{array}$ & $\begin{array}{l}0.249^{* *} \\
(0.067)\end{array}$ & $\begin{array}{l}0.276^{* *} \\
(0.067)\end{array}$ & $\begin{array}{l}0.718^{* *} \\
(0.069)\end{array}$ & $\begin{array}{l}0.638^{* *} \\
(0.070)\end{array}$ & $\begin{array}{l}0.618^{* *} \\
(0.070)\end{array}$ \\
\hline Light Mexicans & $\begin{array}{l}0.427^{* *} \\
(0.049)\end{array}$ & $\begin{array}{l}0.382^{* *} \\
(0.051)\end{array}$ & $\begin{array}{l}0.369^{* *} \\
(0.051)\end{array}$ & $\begin{array}{l}0.580^{* *} \\
(0.051)\end{array}$ & $\begin{array}{l}0.485^{* *} \\
(0.053)\end{array}$ & $\begin{array}{l}0.461^{* *} \\
(0.054)\end{array}$ \\
\hline Light Puerto Ricans & $\begin{array}{l}0.470^{* *} \\
(0.057)\end{array}$ & $\begin{array}{l}0.393^{* *} \\
(0.059)\end{array}$ & $\begin{array}{l}0.360^{* *} \\
(0.059)\end{array}$ & $\begin{array}{l}0.483^{* *} \\
(0.059)\end{array}$ & $\begin{array}{l}0.378^{* *} \\
(0.061)\end{array}$ & $\begin{array}{l}0.359^{* *} \\
(0.062)\end{array}$ \\
\hline Light Cubans & $\begin{array}{l}0.186^{* *} \\
(0.050)\end{array}$ & $\begin{array}{l}0.162^{* *} \\
(0.051)\end{array}$ & $\begin{array}{l}0.186^{* *} \\
(0.051)\end{array}$ & $\begin{array}{l}0.613^{* *} \\
(0.053)\end{array}$ & $\begin{array}{l}0.575^{* *} \\
(0.053)\end{array}$ & $\begin{array}{l}0.557^{* * *} \\
(0.054)\end{array}$ \\
\hline Whites (omitted) & & & & & & \\
\hline Constant & 2.123 & 2.347 & 2.385 & 1.916 & 2.297 & 2.455 \\
\hline$R^{2}$ & .058 & .069 & .076 & .073 & .088 & .099 \\
\hline$R^{2}$ change & - & $.011^{* *}$ & $.007^{* *}$ & - & $.015^{* *}$ & $.011^{* *}$ \\
\hline
\end{tabular}

Note. Estimated coefficients in Model 2 are adjusted for gender, age, income, education, nativity, and acculturation. Estimated coefficients in Model 3 are adjusted for all the variables in Model 2 as well as political ideology, party affiliation, and love for the United States (coefficients not shown).

${ }^{* *} p \leq .01$ (two-tailed tests).

complexion. Fig. 3 also shows that only 7 in 10 Cubans, a little more than 4 in 10 Puerto Ricans, and a third of Mexicans are categorized as having a light complexion. Because there are real differences by ethnicity in skin tone, in the next set of analyses we combine Latino ethnicity and skin color into one variable. ${ }^{14}$ By coding Latinos by both ethnicity and skin color we will be able to assess whether or not dark, medium, and light Mexicans, Puerto Ricans, and Cubans differ from one another, differ from those within their ethnic group of a different skin color, as well as whether they differ from Blacks and Whites on these social attitude measures.

Do the social attitudes of the groups we study differ by race/ethnicity and skin color, with Blacks and Whites representing the polarities and some Latinos somewhere in the middle and others closer to Whites and Blacks based on skin color? Responses to the two questions on support for increased government help for Blacks and legal immigrants, reported in Table 1, indicate a more complicated portrait. For instance, Black and White attitudes do not represent the polarities in attitudes that one might expect. To be sure, Blacks are more likely to endorse 
Table 3

Gross and net effect of race/ethnicity and skin color on perceptions of discrimination against Blacks and affect toward Whites and Blacks (standard errors in parentheses)

\begin{tabular}{|c|c|c|c|c|c|c|c|c|c|}
\hline & \multicolumn{3}{|c|}{ Discrimination against Blacks } & \multicolumn{3}{|c|}{ Warmth toward Whites } & \multicolumn{3}{|c|}{ Warmth toward Blacks } \\
\hline & Model 1 & Model 2 & Model 3 & Model 1 & Model 2 & Model 3 & Model 1 & Model 2 & Model 3 \\
\hline \multicolumn{10}{|l|}{ Race/ethnicity } \\
\hline Blacks & $\begin{array}{r}0.122 \\
(0.186)\end{array}$ & $\begin{array}{r}0.042 \\
(0.177)\end{array}$ & $\begin{array}{r}-0.069 \\
(0.177)\end{array}$ & $\begin{array}{r}-20.082^{* *} \\
(4.596)\end{array}$ & $\begin{array}{r}-18.993^{* *} \\
(4.537)\end{array}$ & $\begin{array}{r}-19.207^{* *} \\
(4.535)\end{array}$ & $\begin{array}{r}14.584^{* *} \\
(4.806)\end{array}$ & $\begin{array}{r}13.464^{* *} \\
(4.709)\end{array}$ & $\begin{array}{r}11.732^{* *} \\
(4.700)\end{array}$ \\
\hline Dark Mexicans & $\begin{array}{r}0.003 \\
(0.086)\end{array}$ & $\begin{array}{c}0.185^{*} \\
(0.086)\end{array}$ & $\begin{array}{c}0.142^{+} \\
(0.086)\end{array}$ & $\begin{array}{r}-9.382^{* *} \\
(2.132)\end{array}$ & $\begin{array}{r}-3.726^{+} \\
(2.202)\end{array}$ & $\begin{array}{l}-3.069 \\
(2.213)\end{array}$ & $\begin{array}{r}-5.727^{* *} \\
(2.229)\end{array}$ & $\begin{array}{r}-1.243 \\
(2.285)\end{array}$ & $\begin{array}{l}-0.957 \\
(2.293)\end{array}$ \\
\hline Dark Puerto Ricans & $\begin{array}{l}0.271^{*} \\
(0.119)\end{array}$ & $\begin{array}{l}0.413^{* *} \\
(0.115)\end{array}$ & $\begin{array}{l}0.387^{* *} \\
(0.115)\end{array}$ & $\begin{array}{r}-13.282^{* *} \\
(2.947)\end{array}$ & $\begin{array}{r}-8.619^{* *} \\
(2.951)\end{array}$ & $\begin{array}{r}-7.201^{* *} \\
(2.956)\end{array}$ & $\begin{array}{r}-11.336^{* *} \\
(3.082)\end{array}$ & $\begin{array}{r}-8.089^{* *} \\
(3.063)\end{array}$ & $\begin{array}{r}-7.178^{*} \\
(3.063)\end{array}$ \\
\hline Dark Cubans & $\begin{array}{r}-0.164 \\
(0.161)\end{array}$ & $\begin{array}{r}0.053 \\
(0.155)\end{array}$ & $\begin{array}{r}0.086 \\
(0.154)\end{array}$ & $\begin{array}{r}-6.688^{+} \\
(4.006)\end{array}$ & $\begin{array}{l}-2.276 \\
(3.985)\end{array}$ & $\begin{array}{r}-1.427 \\
(3.971)\end{array}$ & $\begin{array}{r}-10.806^{* *} \\
(4.189)\end{array}$ & $\begin{array}{r}-6.995^{+} \\
(4.135)\end{array}$ & $\begin{array}{r}-7.139^{+} \\
(4.114)\end{array}$ \\
\hline Medium Mexicans & $\begin{array}{l}-0.074 \\
(0.075)\end{array}$ & $\begin{array}{r}0.075 \\
(0.075)\end{array}$ & $\begin{array}{r}0.059 \\
(0.075)\end{array}$ & $\begin{array}{r}-8.592^{* *} \\
(1.864)\end{array}$ & $\begin{array}{r}-3.364^{+} \\
(1.937)\end{array}$ & $\begin{array}{l}-2.639 \\
(1.936)\end{array}$ & $\begin{array}{r}-7.214^{* *} \\
(1.949)\end{array}$ & $\begin{array}{l}-2.806 \\
(2.010)\end{array}$ & $\begin{array}{l}-2.406 \\
(2.006)\end{array}$ \\
\hline Medium Puerto Ricans & $\begin{array}{l}0.244^{* *} \\
(0.092)\end{array}$ & $\begin{array}{l}0.428^{* *} \\
(0.091)\end{array}$ & $\begin{array}{c}0.372^{* *} \\
(0.091)\end{array}$ & $\begin{array}{r}-13.519^{* *} \\
(2.284)\end{array}$ & $\begin{array}{r}-7.893^{* *} \\
(2.335)\end{array}$ & $\begin{array}{r}-7.163^{* *} \\
(2.354)\end{array}$ & $\begin{array}{r}-5.097^{*} \\
(2.389)\end{array}$ & $\begin{array}{l}-0.890 \\
(2.423)\end{array}$ & $\begin{array}{l}-0.926 \\
(2.439)\end{array}$ \\
\hline Medium Cubans & $\begin{array}{r}-0.620^{* *} \\
(0.103)\end{array}$ & $\begin{array}{r}-0.387^{* *} \\
(0.100)\end{array}$ & $\begin{array}{r}-0.318^{* *} \\
(0.100)\end{array}$ & $\begin{array}{r}-9.132^{* *} \\
(2.568)\end{array}$ & $\begin{array}{r}-5.270^{*} \\
(2.578)\end{array}$ & $\begin{array}{r}-5.364^{*} \\
(2.583)\end{array}$ & $\begin{array}{r}-10.478^{* *} \\
(2.685)\end{array}$ & $\begin{array}{r}-6.495^{* *} \\
(2.675)\end{array}$ & $\begin{array}{r}-5.329^{*} \\
(2.677)\end{array}$ \\
\hline Light Mexicans & $\begin{array}{r}-0.068 \\
(0.077)\end{array}$ & $\begin{array}{r}0.040 \\
(0.077)\end{array}$ & $\begin{array}{r}0.025 \\
(0.077)\end{array}$ & $\begin{array}{r}-5.271^{* *} \\
(1.910)\end{array}$ & $\begin{array}{l}-0.366 \\
(1.971)\end{array}$ & $\begin{array}{r}0.474 \\
(1.976)\end{array}$ & $\begin{array}{r}-5.710^{* *} \\
(1.998)\end{array}$ & $\begin{array}{l}-1.717 \\
(2.045)\end{array}$ & $\begin{array}{l}-0.857 \\
(2.047)\end{array}$ \\
\hline Light Puerto Ricans & $\begin{array}{l}0.273^{* *} \\
(0.089)\end{array}$ & $\begin{array}{l}0.387^{* *} \\
(0.088)\end{array}$ & $\begin{array}{l}0.338^{* *} \\
(0.089)\end{array}$ & $\begin{array}{r}-9.109^{* *} \\
(2.210)\end{array}$ & $\begin{array}{r}-3.472 \\
(2.268)\end{array}$ & $\begin{array}{l}-2.601 \\
(2.283)\end{array}$ & $\begin{array}{r}-4.756^{*} \\
(2.311)\end{array}$ & $\begin{array}{l}-1.621 \\
(2.353)\end{array}$ & $\begin{array}{r}-1.493 \\
(2.366)\end{array}$ \\
\hline Light Cubans & $\begin{array}{r}-0.584^{* *} \\
(0.079)\end{array}$ & $\begin{array}{r}-0.455^{* *} \\
(0.076)\end{array}$ & $\begin{array}{r}-0.379^{* *} \\
(0.077)\end{array}$ & $\begin{array}{l}-3.029 \\
(1.965)\end{array}$ & $\begin{array}{c}-0.731 \\
(1.957)\end{array}$ & $\begin{array}{r}-0.870 \\
(1.975)\end{array}$ & $\begin{array}{r}-10.012^{* *} \\
(2.055)\end{array}$ & $\begin{array}{r}-7.144^{* *} \\
(2.031)\end{array}$ & $\begin{array}{r}-6.265^{* *} \\
(2.046)\end{array}$ \\
\hline Whites (omitted) & & & & & & & & & \\
\hline Constant & 3.162 & 2.962 & 2.701 & 79.834 & 59.758 & 53.456 & 67.249 & 56.361 & 49.363 \\
\hline$R^{2}$ & .082 & .167 & .187 & .026 & .055 & .071 & .020 & .064 & .082 \\
\hline$R^{2}$ change & - & $.085^{* *}$ & $.020^{* *}$ & - & $.029 * *$ & $.016^{* *}$ & - & $.044^{* *}$ & $.018^{* *}$ \\
\hline
\end{tabular}

Note. Estimated coefficients in Model 2 are adjusted for gender, age, income, education, nativity, and acculturation. Estimated coefficients in Model 3 are adjusted for all the variables in Model 2 as well as political ideology, party affiliation, and love for the United States (coefficients not shown).

${ }^{+} p \leq .10$ (two-tailed tests).

$* p \leq .05$ (two-tailed tests).

${ }^{* *} p \leq .01$ (two-tailed tests). 
government assistance for Blacks than are Whites (54\% vs. 24\%, respectively). Dark-skinned Mexicans (61\%), Puerto Ricans (65\%), and Cubans (70\%), however, are more likely than anyone, including non-Hispanic Blacks (54\%), to endorse increased government assistance for Blacks. Among Puerto Ricans it is those coded as having a medium complexion (compared to dark and light skin Puerto Ricans) that are most likely to endorse increased government help for Blacks. Dark-skinned Cubans (70\%) support government assistance for Blacks more than medium (48\%) and light skin Cubans (41\%). There are no skin color differences among Mexicans in their attitudes toward increased government help for Blacks. However, all Latinos are more likely to endorse increased government help for Blacks than non-Hispanic Whites.

When we examine responses to the question concerning attitudes toward increased government assistance for legal immigrants and refugees, we see a similar pattern. Whites are least likely to endorse greater government help for legal immigrants and refugees (19\%) followed next by Blacks (29\%). The Black-White difference remains but is much more muted than for the previous question. Among all three Latino groups there are differences in support for increased government help for legal immigrants by skin color. Darker skinned Latinos are more supportive of government assistance than light skin Latinos. These skin color differences are especially pronounced among Cubans: $83 \%$ of dark skin Cubans, $64 \%$ of medium skin Cubans, and 55\% of light skin Cubans endorse increased spending on behalf of legal immigrants and refugees.

Examining responses to the question concerning perceived discrimination against Blacks, we see in Table 1, similar to other research, that there is a rather large gap between Blacks and Whites in their perception of discrimination against Blacks (see Bobo \& Suh, 2000; Forman, 1997; Kluegel \& Bobo, 2001). For instance, Blacks are twice as likely as Whites to perceive "a lot" of discrimination against Blacks (63 and 32\%, respectively). Further, Mexicans, Puerto Ricans, and Cubans responses reveal a clear racial hierarchy in views about discrimination against Blacks: Mexicans and Puerto Ricans regardless of skin color are more likely than non-Hispanic Whites to perceive "a lot" of discrimination against Blacks, whereas dark skin Cubans view the same amount of discrimination against Blacks as non-Hispanic Whites, and medium as well as light skin Cubans view less discrimination against Blacks than non-Hispanic Whites.

The final two questions ask respondents about their feelings toward Whites and Blacks. As one might expect, non-Hispanic Whites express the most warmth toward Whites. Skin color does not differentiate between Mexicans and Cubans positive affect for Whites. Only dark and medium skin color Puerto Ricans report lower feelings of warmth toward Whites comparable to those of Blacks. A similar pattern is observed for feelings toward non-Hispanic Blacks. The largest differences are between Blacks and Whites. Regardless of skin color each Latino group is closer to non-Hispanic Whites in their expressed feelings toward Blacks than they are to non-Hispanic Blacks.

Overall, for three of the five questions most Latinos appear to occupy a distinct category from Blacks and Whites in their attitudes toward race-related issues. Dark and medium complected Puerto Ricans, whose attitudes are consistently closer to those of Blacks, are an exception to this pattern. In all, these data provide suggestive evidence in support of the Latin Americanization thesis - as it pertains to social attitudes there does appear to be a three-tier structure 
forming with many Latinos fitting into a distinct category from both Whites and Blacks. Nevertheless, these initial descriptive results must be read with caution. Individuals' attitudes toward race-related issues are shaped not only by there race/ethnicity and skin color but a range of other factors. For example, it is possible that the differences in race-related issues apparent in Table 1 may actually reflect group differences in social background factors such as age, gender, income, education, acculturation, nativity and/or group differences in political values and ideology (political orientation). To investigate this possibility, we estimate a series of regression models reported in Tables 2 and 3. Model 1 of Tables 2 and 3 includes only a set of dummy variables identifying Blacks, dark skin, medium skin, and light skin Mexicans, Puerto Ricans, and Cubans (with White respondents as the omitted category). In Models 2 and 3 of Tables 2 and 3 we sequentially add social background factors and political orientation measures. We can get a sense of the extent to which these additional variables explain a portion (or possibly all) of the apparent skin color and race/ethnicity differences in race-related attitudes by comparing the magnitude and statistical significance of the race/ethnicity and skin color coefficients in Model 1 to the same coefficients reported in Model 2 after controlling for social background factors and Model 3 after controlling for political values and ideology.

In general, the regression coefficients reported in Table 2 indicate that differences in social background factors and political orientation do not explain the race/ethnicity and skin color differences in attitudes toward government help for Blacks and government help for legal immigrants and refugees. ${ }^{15}$ Each estimated model in Table 2 shows a statistically significant effect for race/ethnicity and skin color. For instance, according to Model 1 each group is significantly different from non-Hispanic Whites in their greater endorsement of increased government assistance for Blacks. These differences seem to be largest for Blacks and dark skin Puerto Ricans and Cubans. In Models 2 and 3, we see that once we control for social background factors and political values and ideology, the influence of race/ethnicity and skin color on increased government assistance for Blacks is slightly reduced. This process of attenuation highlights that the relationship between race/ethnicity and skin color and increased government assistance for Blacks is only marginally explained by taking into account factors like gender, age, income, education, acculturation, nativity, and political orientation. Although the variation in government help for Blacks explained by these variables is low (7.6\%, see Model 3) it is important to note that a simple control for race/ethnicity and skin color accounts for $76 \%$ of this variation.

A similar pattern is observed for increased government assistance for legal immigrants and refugees. Model 1 in Table 2 shows that Blacks are the only group that are similar to Whites in their views about government assistance for legal immigrants. All Latinos, irrespective of skin tone, appear to endorse greater governmental support for immigrants compared to Whites. As one might imagine given their history, Cubans of all skin colors appear to give the strongest endorsement to government assistant for legal immigrants and thus have the largest differences from Whites. These patterns persist after controls for background characteristics and political views. Again, race/ethnicity and skin color accounts for a substantial portion $(74 \%)$ of the explained variation in government assistance for legal immigrants.

Table 3 reports the results for perceived discrimination against Blacks and warmth toward Whites and Blacks. The results for perceived discrimination are quite intriguing. Puerto Ricans, of all hews, significantly differ from Whites in their assessment of the amount of discrimination 
experienced by Blacks. Puerto Ricans believe there is more discrimination against Blacks compared to Whites. In contrast, medium and light complected Cubans believe there is less discrimination against Blacks than do Whites. These patterns cannot be attributed to a larger number of Puerto Ricans compared to Cubans identifying as "Black" (recall, 4\% of Puerto Ricans and $3 \%$ of Cubans self-identity as "Black" in our sample, see Fig. 2). According to Model 2, once we adjust for social background characteristics, dark skin Mexicans are more likely than Whites to believe that Blacks experience discrimination. Comparing the coefficients across the three models we see some reductions. Despite these reductions in the unstandardized regression coefficients for race/ethnicity and skin color, they by and large remain statistically significant.

As one might expect, Blacks followed by dark and medium complected Mexicans and all Puerto Ricans differed most from Whites in feelings of warmth toward Whites. In general, however, all groups with the exception of light skin Cubans feel less warmth toward Whites compared with non-Hispanic Whites. Once we control for social background factors in Model 2 , we see that some of the differences disappear completely or become much more muted. These pattern of results imply that race/ethnicity and skin color differences in affect for Whites can be partially explained by average differences among these groups in their distributions on certain background factors. Model 3 shows the race/ethnicity coefficients after controlling for several measures of political orientation. These results indicate that taking into account political values and ideology produces appreciable changes in the influence of race/ethnicity and skin color on affect toward Whites, compared to those observed in Models 1 and 2. Clearly, a non-trivial portion of the influence of race/ethnicity and skin color on warmth toward Whites is a direct manifestation of ethnicity and skin color differences in social background characteristics and political orientation. In fact, only $37 \%$ of the variation in affect for Whites $(7.1 \%)$ is accounted for by race/ethnicity and skin color.

There are a number of striking patterns in Model 1 for affect toward Blacks. First, as would be expected, Blacks substantially differ from Whites in their affect toward Blacks. Second, Latinos of all hews express less warmth toward Blacks than non-Hispanic Whites. Third, Cubans of all skin colors differ most (dark Puerto Ricans are the one exception to this pattern) from Whites in their more negative feelings toward Blacks. Fourth, dark skin Puerto Ricans are more negative in their feelings toward Blacks than medium and light skin Puerto Ricans. Controls introduced in Model 2 indicate that some of these differences are attributable to group differences in social background characteristics. In fact, half of the coefficients that were statistically significant in Model 1 are no longer so in Model 2. Model 3 reveals that the race/ethnic and skin color differences that remain are not related to differences in political orientation.

\section{Summary and conclusion}

The main purpose of this study was to investigate several aspects of the Latin Americanization thesis. First, we found mixed results for our question as to whether there are intragroup differences among Latinos in attitudes toward race-related issues associated with skin color. Overall there appears to be much more variation in attitudes by skin color for Puerto Ricans and Cubans than for Mexicans. This is not entirely surprising given that the former have differing 
racial histories and hierarchies that include not only White and Black categories (e.g., blancos and negros) but middle categories (e.g., trigueño and moreno) (Rodriguez, 2000). While there is skin color variation within the Mexican origin population overall, the absence of a history of African presence and thus lack of a "Black" category may mean that Mexican-American's experiences in the U.S. differ less from one another than for instance dark versus light Cubans differ from one another.

With regard to our second set of questions as to whether and how different Latino ethnic groups seem to fit into a three-tier racial hierarchy, we also find mixed results. For instance, we found that both ethnicity and skin color matter for Latinos' social attitudes and that some of the groups (e.g., Mexicans, medium complected Puerto Ricans and Cubans) seem to occupy a clear middle category between Whites and Blacks. Darker complected Puerto Ricans seem more similar to non-Hispanic Blacks. However, there are issues in which they are more similar to other Latinos (e.g., attitudes toward immigration). Moreover, our multivariate regression models further suggest that skin color variation is more relevant for Puerto Ricans overall than it is for either Cuban and Mexicans who tend to be more similar to each other within ethnic group and also more similar to Whites. The findings from the multivariate regression models also show that at least some of the ethnic and skin color variation in attitudes is explained by the differing social and demographic characteristics of each group.

Overall, this study examined just one aspect of the Latin Americanization thesis. While the overall theory captures patterns in group experience and social location, we deal with the ways in which groups take up their positioning - specifically their attitudes on race-related issues. We find evidence that there is movement towards a three-tiered division with many Latinos differing substantially from both Whites and Blacks. Moreover, we find that there is great variation within Latinos by both ethnicity and skin color. Thus, it may well be true in the coming years that different Latino groups will come to occupy very different spaces within the racial hierarchy. There also seems to be some evidence, however, that there remain issues which unite Latinos (e.g., attitudes about immigration) that may keep future racial categories from attaining stability and/or permanence.

Despite the fact that we found mixed support for the Latin Americanization thesis, we believe it is an important and timely idea to further explore as the U.S. continues to experience substantial demographic changes. Discovering more about the attitudes of often-excluded racial groups will become more significant as these changes occur. Future studies may further examine the power and usefulness of the Latin Americanization thesis. For instance, other datasets may enable the examination of groups not present in our study including Asian Americans, and other Latino groups (e.g., Dominicans, Guatemalans, Nicaraguans, and Salvadorians). Moreover, other datasets may provide clearer information on how different generations of Asian and Latinos fit into the tri-racial stratification system (e.g., differences between firstand second-generation Latinos). Typically, first-generation individuals are likely to express attitudes that differ from second-generation Latinos since the first-generation often live in ethnic enclaves which provide distance from most other racial and ethnic groups (see Forman, 2004; Murguia \& Forman, 2003). Also, studies are needed that examine bi-racial individuals' racial attitudes and status in the U.S. racial hierarchy.

In sum, our analysis of Blacks', Whites', and Latinos' social attitudes indicate that there is a three-tier structure forming, and that where Latinos appear to fall depends, in part, on their 
ethnicity and in part, on their skin color (this is especially true for Puerto Ricans). Ultimately, further research is needed to more fully investigate the overall viability of the Latin Americanization thesis, but for now it appears to hold promise for understanding the future of race relations.

\section{Notes}

1. Throughout this article we frequently use the panethnic label of "Latino" or "Asian." We recognize that both Asians and Latinos are diverse groups and that treating them as a single category may not provide an accurate representation of important subgroups (for a discussion of panethnic labels and Latino identity see DeSipio, 1996; Lopez \& Espiritu, 1990). To this end, in our empirical analysis we give attention to specific subgroups (i.e., Mexicans, Puerto Ricans, and Cubans).

2. It is important to note that recent historiography has challenged the conventional wisdom that Italians were "non-White" and then became "White" (see Guglielmo, 2003).

3. In this article we use the term "White" to refer to non-Hispanic Whites and "Black" to refer to non-Hispanic Blacks. We use these terms interchangeably in the text.

4. Data on Whites' neighborhood preferences also mirror this rank ordering in residential segregation patterns (see Bobo \& Zubrinksy, 1996).

5. Which pathway immigrants follow is directly related to the social and economic capital that immigrants have access to when they arrive in the U.S. For some scholars, social capital is viewed as the critical dimension in understanding the distinctive assimilation processes experienced by recent immigrants (Portes \& Zhou, 1993). Portes and Zhou (1993) argue that it is the strength or weakness of the ethnic specific social networks that immigrants rely upon for access to employment, housing, and a variety of other opportunities in the host society that explain the divergent destinies of recent immigrants. Those who arrive in the U.S. with strong social networks are able to successfully experience some social and economic advancement whereas those arriving with weak social networks have greater difficulty assimilating into mainstream white society and in all likelihood assimilate into the lowest stratum of U.S. society where there is little opportunity for social and economic advancement. Under-theorized in this framework is the role that race and skin color play in shaping the opportunity structure available to recent immigrants and their children. The "color line" has been an important feature of the U.S. social and political landscape and thus the role of race/ethnicity and skin color must be front and center in any theorizing of recent immigrants assimilation and acculturation (see Almaguer, 1994; Murguia \& Forman, 2003; Saito, 1998). For many Black, Asian, and Latino immigrants phenotype and skin color in particular are critical factors in shaping their experience in the United States (see Allen, Telles, \& Hunter, 2000; Freeman, 1999; Murguia \& Forman, 2003; Tuan, 1998). In fact, many of these groups report experiencing discrimination and it has been shown that this has important implications for their well-being (see Amaro, Russo, \& Johnson, 1987; Forman, 2003; Forman, Williams, \& Jackson, 1997; Kuo, 1995; Noh, Beiser, Kaspar, Hou, \& Rummens, 1999; Rumbaut, 1994; Salgado de Snyder, 1987; Tuan, 1998). 
6. Fig. 1 should be viewed as a heuristic model and as a first approximation to the idea of a three-tier racial hierarchy in the United States.

7. This group is referred to as Marielitos and they were more likely to be non-White than previous Cuban refugees (see Aguirre, Saenz, \& James, 1997).

8. Shade discrimination refers to the rank ordering of individuals based upon phenotype (e.g., skin color, facial characteristics, and hair texture) (see Kinsbrunner, 1996). One aspect of phenotype that previous research has revealed is powerfully linked to the sorting of educational and labor market opportunities among Blacks and Latinos is skin color (see Allen et al., 2000; Arce, Murguia, \& Frisbie, 1987; Keith \& Herring, 1991; Murguia \& Forman, 2003; Murguia \& Telles, 1996; Telles \& Lam, 1998; Telles \& Murguia, 1990).

9. Here we use white supremacy to refer to the social and political regimes that emerged in the 16th century and have consistently reinvented themselves globally to sustain the position of those racialized as "White" (see Bonilla-Silva, 2001, 2003; Mills, 1997, 1998, 2000).

10. Although the Latin Americanization thesis discusses both Asians and Latinos, in this study we have chosen to focus on Latinos. We focus here on Latinos because the National Latino Political Survey had too few Asian respondents to reliably study them.

11. "No opinion" was also a possible response category. Approximately, 5\% of the sample chose this response category. Further, there were no race/ethnic differences in "no opinion" responses: Mexicans (6\%), Puerto Ricans (5\%), Cubans (6\%), Whites (4\%), and Blacks (4\%). Because the "no opinion" response category was small we combined the categories "no opinion" and "left the same." To examine whether our results were sensitive to this coding scheme we conducted additional analyses in which we compared results from combining the two response categories to separating them out. The results from these analyses were virtually identical so we combined the two categories.

12. Following Aguirre and Saenz (2002) we divided the number of years a respondent has lived in the United States by their current age.

13. Two of the three dependent variables used in our study are ordinal with limited response categories. It is generally understood that when your dependent variable is ordinal with limited response categories it is more appropriate to estimate statistical models using maximum likelihood ordered logit regression models (see Hosmer \& Lemeshow, 1989; Winship \& Mare, 1984); however, in this study we decided to present the ordinary least squares estimates because of their straightforward interpretation. To check whether our parameter estimates were sensitive to the statistical method used, in analyses not shown here, we also estimated our regression models using the ordered logit regression procedure. The results from these analyses did not differ substantively from those presented. These results are available from the authors.

14. Ideally we would have also classified non-Hispanic Blacks in the same manner that we coded Latino respondents, however, interviewers did not assess Blacks' skin color.

15. Although several variables are controlled for in these estimated regression models we report and focus our discussion on the race/ethnicity and skin color coefficients because we are centrally interested in testing the Latin Americanization thesis. 


\section{Acknowledgments}

This article was completed while the first and third author were Visiting Fellows at the Research Institute of Comparative Studies in Race and Ethnicity (RICSRE) at Stanford University. We gratefully acknowledge the support and hospitality that the Institute provided. We thank the Institute for Research on Race and Public Policy at the University of Illinois at Chicago for providing critical seed funds to support this research in its early stages. We also express our appreciation to Melissa Martin for assistance in the preparation of tables and figures. Finally, we acknowledge the helpful comments of Eduardo Bonilla-Silva and Jennifer Hamer on an earlier version of this manuscript. The authors equally contributed to this article.

\section{References}

Aguirre, B., \& Saenz, R. (2002). The naturalization of Mexican and Cuban immigrants in the US: Testing the effects of collectively expected durations of migration. International Migration Review, 36 (1), $103-124$.

Aguirre, B., Saenz, R., \& James, B. (1997). Marielitos ten years later: The scarface legacy. Social Science Quarterly, 78 (2), 487-507.

Alba, R., \& Nee, V. (1997). Rethinking assimilation theory for a new era of immigration. International Migration Review, 31 (4), 826-874.

Allen, W., Telles, E., \& Hunter, M. (2000). Skin color, income, and education: A comparison of African Americans and Mexican Americans. National Journal of Sociology, 12 (1), 129-180.

Almaguer, T. (1994). Racial fault lines. Berkeley, CA: University of California Press.

Amaro, H., Russo, N., \& Johnson, J. (1987). Family and work predictors of psychological well-being among Hispanic women professionals. Psychology of Women Quarterly, 11, 505-521.

Arce, C., Murguia, E., \& Frisbie, W. P. (1987). Phenotype and life chances among Chicanos. Hispanic Journal of Behavioral Sciences, 9 (1), 19-32.

Bobo, L., \& Hutchings, V. (1996). Perceptions of racial group competition: Extending Blumer's theory of group position to a multiracial social context. American Sociological Review, 61 (6), 951-972.

Bobo, L., \& Suh, S. (2000). Surveying racial discrimination: Analyses from a multiethnic labor market. In L. Bobo, M. Oliver, J. Johnson, \& A. Valenzuela (Eds.), Prismatic metropolis (pp. 527-564). New York, NY: Russell Sage Foundation.

Bobo, L., \& Zubrinksy, C. (1996). Attitudes on residential integration: Perceived status differences, mere in-group preference, or racial prejudice? Social Forces, 74, 883-909.

Bonilla-Silva, E. (2001). White supremacy \& racism in the post-civil rights era. Boulder, CO: Lynne Rienner.

Bonilla-Silva, E. (2003). Racism without racists. Lanham, MD: Rowman \& Littlefield.

Bonilla-Silva, E., Forman, T., Lewis, A., \& Embrick, D. (2003). It wasn't me: How will race and racism work in 21 st century America. Research in Political Sociology, 12, 111-134.

Degler, C. (1971). Neither black nor white: Slavery and race relations in Brazil and the United States. New York, NY: The Macmillan Company.

de la Garza, R., DeSipio, L., Garcia, F. C., Garcia, J., \& Falcon, A. (1992). Latino voices: Mexican, Puerto Rican, and Cuban perspectives on American politics. Boulder, CO: Westview Press.

de la Garza, R., Falcon, A., \& Garcia, F. C. (1996). Will the 'real' Americans please stand up: Anglo and Mexican American support of core American political values. American Journal of Political Science, 40, 335-351.

DeSipio, L. (1996). More than the sum of its parts: The building blocks of a pan-ethnic Latino identity. In W. Rich (Ed.), The politics of minority coalitions (pp. 177-189). Westport, CT: Praeger.

Farley, R. (1996). The new American reality. New York, NY: Russell Sage Foundation.

Fields, B. (2001). Whiteness, racism, and identity. International Labor and Working-Class History, 60, 48-56.

Forman, T. (2001). Social change, social context, and White youth's racial attitudes. Ph.D. Dissertation, Department of Sociology, University of Michigan, Ann Arbor, Michigan. 
Forman, T. (2003). The social psychological costs of racial segmentation in the workplace: A study of African Americans' well being. Journal of Health and Social Behavior, 44 (3), 332-352.

Forman, T. (2004). Revisiting the immigrant hypothesis: Latinos' attitudes toward Blacks and Asians. Unpublished manuscript, Department of Sociology, University of Illinois at Chicago.

Forman, T. (in press). Color-blind racism and racial indifference: The role of racial apathy in facilitating enduring inequalities. In M. Krysan \& A. Lewis (Eds.), Changing terrain of race and ethnicity. New York, NY: Russell Sage Foundation.

Forman, T., Williams, D., \& Jackson, J. (1997). Race, place, and discrimination. Perspectives on Social Problems, 9, 231-261.

Freeman, L. (1999). A note on the influence of African heritage on ssegregation: The case of Dominicans. Urban Affairs Review, 35 (1), 137-146.

Gans, H. (1999). The possibility of a new racial hierarchy in the twenty-first century United States. In M. Lamont (Ed.), The cultural territories of race (pp. 371-390). Chicago, IL: University of Chicago Press.

Gordon, L. (1997). Her Majesty's other children. New York, NY: Rowman and Littlefield.

Gordon, M. (1964). Assimilation in American life. The role of race, religion, and national origin. New York, NY: Oxford University Press.

Guglielmo, T. (2003). White on arrival: Italians, race, color, and power in Chicago, 1890-1945. New York, NY: Oxford University Press.

Hosmer, D., \& Lemeshow, S. (1989). Applied logistic regression. New York, NY: Wiley.

Jackman, M., \& Crane, M. (1986). "Some of my best friends are Black ...": Interracial friendship and Whites' racial attitudes. Public Opinion Quarterly, 50, 459-486.

Keith, V., \& Herring, C. (1991). Skin tone and stratification in the Black community. American Journal of Sociology, 97 (30), 760-778.

Kinsbrunner, J. (1996). Not of pure blood: The free people of color and racial prejudice in nineteenth-century Puerto Rico. Durham: Duke University Press.

Kluegel, J., \& Bobo, L. (2001). Perceived group discrimination and policy attitudes. In A. O'Connor, C. Tilly, \& L. Bobo (Eds.), Urban inequality (pp. 163-198). New York, NY: Russell Sage Foundation.

Krysan, M. (1998). Privacy and the expression of White racial attitudes: A comparison across three contexts. Public Opinion Quarterly, 62, 506-544.

Kuo, W. (1995). Coping with racial discrimination: The case of Asian Americans. Ethnic and Racial Studies, 18 (1), 109-127.

Lewis, A. (2003). Race in the schoolyard: Negotiating the color line in classrooms and communities. New Brunswick, NJ: Rutgers University.

Lind, M. (1998, August 16). The beige and the Black. New York Times Magazine, pp. 38-39.

Lopez, D., \& Espiritu, Y. (1990). Panethnicity in the United States: A theoretical framework. Ethnic and Racial Studies, 13 (2), 198-224.

Massey, D., \& Fischer, M. (1999). Does rising income bring integration? New results for Blacks, Hispanics, and Asians in 1990. Social Science Research, 28 (3), 316-326.

McDaniel, A. (1995). The dynamics of racial composition in the United States. Daedalus, 124, 179-198.

McKee, J. B. (1993). Sociology and the race problem: The failure of a perspective. Urbana, IL: University of Illinois Press.

Mills, C. (1997). The racial contract. Ithaca, NY: Cornell University Press.

Mills, C. (1998). Blackness visible. Ithaca, NY: Cornell University Press.

Mills, C. (2000). Race and the social contract tradition. Social Identities, 6 (4), 441-462.

Murguia, E., \& Forman, T. (2003). Shades of whiteness: The Mexican American experience in relation to Anglos and Blacks. In A. Doane \& E. Bonilla-Silva (Eds.), Whiteout: The continuing significance of racism (pp. 63-79). New York, NY: Routledge.

Murguia, E., \& Telles, E. (1996). Phenotype and schooling among Mexican Americans. Sociology of Education, 69 (4), 276-289.

Noh, S., Beiser, M., Kaspar, V., Hou, F., \& Rummens, J. (1999). Perceived racial discrimination, depression, and coping: A study of southeast Asian refugees in Canada. Journal of Health and Social Behavior, 40 (3), 193-207. 
Portes, A., \& Zhou, M. (1993). The new second generation: Segmented assimilation and its variants. Annals of the American Academy of Political and Social Science, 530, 74-96.

Rodriguez, C. (1992). Race, culture, and Latino 'otherness' in the 1980 Census. Social Science Quarterly, 73 (4), 930-937.

Rodriguez, C. (2000). Changing race: Latinos, the census, and the history of ethnicity in the United States. New York, NY: New York University Press.

Rodriguez, C., \& Cordero-Guzman, H. (1992). Placing race in context. Ethnic and Racial Studies, 15 (4), 523-542.

Rumbaut, R. (1994). The crucible within: Ethnic identity, self-esteem, and segmented assimilation among children of immigrants. International Migration Review, 28 (4), 748-794.

Saito, L. (1998). Race and politics: Asian Americans, Latinos, and Whites in a Los Angeles suburb. Urbana, IL: University of Illinois Press.

Salgado de Snyder, V. N. (1987). Factors associated with acculturative stress and depressive symptomatology among married Mexican immigrant women. Psychology of Women Quarterly, 11, 475-488.

Sears, D., Van Laar, C., Carrillo, M., \& Kosterman, R. (1997). Is it really racism?: The origins of White Americans' opposition to race-targeted policies. Public Opinion Quarterly, 61 (1), 16-53.

Suarez-Orozco, M. (2000). Everything you ever wanted to know about assimilation but were afraid to ask. Daedalus, 129 (40), 1-30.

Telles, E., \& Lam, N. (1998). Does it matter who answers the race question? Racial classification and income inequality in Brazil. Demography, 35 (4), 465-474.

Telles, E., \& Murguia, E. (1990). Phenotypic discrimination and income differences among Mexican Americans. Social Science Quarterly, 71 (4), 682-696.

Tuan, M. (1998). Forever foreigners or honorary Whites?: The Asian ethnic experience today. New Brunswick, NJ: Rutgers University Press.

U.S. Bureau of the Census. (2001). Statistical abstract of the United States. Washington, DC: Government Printing Office.

Warren, J., \& Twine, F. W. (1997). White Americans, the new minority? Non-Blacks and the ever-expanding boundaries of whiteness. Journal of Black Studies, 28 (2), 200-218.

Welch, S., \& Sigelman, L. (2000). Getting to know you? Latino-Anglo social contact. Social Science Quarterly, $81(1), 67-83$.

Winship, C., \& Mare, R. (1984). Regression models with ordinal variables. American Sociological Review, 49 (4), 512-525.

Yancey, G. (2003). Who is White? Latinos, Asians, and the new Black/NonBlack divide. Boulder, CO: Lynne Rienner.

Zhou, M. (1997). Segmented assimilation: Issues, controversies, and recent research on the new second generation. International Migration Review, 31 (4), 975-1008. 